\title{
Evaluating the clinical significance of nontuberculous mycobacteria isolated from respiratory samples in Iran: an often overlooked disease
}

This article was published in the following Dove Press journal:

Infection and Drug Resistance

\author{
Zahra Mortazavi' \\ Ahmadreza Bahrmand ${ }^{2}$ \\ Fatemeh Sakhaee ${ }^{2}$ \\ Reza Hosseini Doust ${ }^{\prime}$ \\ Farzam Vaziri ${ }^{2,3}$ \\ Seyed Davar Siadat ${ }^{2,3}$ \\ Abolfazl Fateh ${ }^{2,3}$ \\ 'Department of Microbiology, Faculty of \\ Advance Science, Tehran Medical Science, \\ Islamic Azad University, Tehran, Iran; \\ ${ }^{2}$ Department of Mycobacteriology and \\ Pulmonary Research, Pasteur Institute of \\ Iran, Tehran, Iran; ${ }^{3}$ Microbiology \\ Research Center (MRC), Pasteur \\ Institute of Iran, Tehran, Iran
}

Purpose: Nontuberculous mycobacteria (NTM) infection is an increasing problem worldwide whose clinical significance is still largely unknown. The aim of this study was to investigate the epidemiology of NTM infection from respiratory samples and to determine their clinical significance.

Patients and methods: This cross-sectional study was performed on 7,825 clinical samples from December 2015 to December 2017. Detection was conducted using phenotypic and genotypic (hsp65 PCR-RFLP, rpoB, and 16S rRNA genes sequencing) methods. All clinical information including symptoms and radiological findings was extracted from patients' records.

Results: A total of 478 were confirmed to have respiratory samples which were culture positive for mycobacteria, with the prevalence of NTM infection obtained as 53 (11.1\%). Overall, Mycobacterium (M.) fortuitum was the most frequent NTM isolate, followed by M. simiae, $M$. kansasii, M. gordonae, and $M$. conceptionense. There was a relationship between NTM isolates and gender $(P=0.039)$, symptoms $(P=0.048)$, and radiographic findings $(P=0.013)$. Bronchiectasis, infiltration, and cavitary lesion were the most frequent radiological findings in $M$. fortuitum, $M$. simiae, and M. kansasii, respectively, with cough being the most frequent symptom.

Conclusion: We reported five different NTM isolates in respiratory samples with a high frequency of M. fortuitum. NTM infections may play an important role in causing pulmonary disease and in tuberculosis management in endemic settings. Nevertheless, more studies are required to further examine the clinical significance of NTM isolates.

Keywords: nontuberculous mycobacteria, respiratory samples, clinical significance, Mycobacterium fortuitum

\section{Introduction}

Nontuberculous mycobacteria (NTM) which can cause pulmonary and extrapulmonary infections in susceptible persons constitute ubiquitous environmental bacteria often found in water and soil. Nowadays, NTM infections are increasingly identified as important causes of morbidity and mortality in patients owing to misdiagnosis and unsuitable treatment. In contrast with tuberculosis (TB) infection, the knowledge about NTM infections is still limited. While $>190$ NTM species have been recognized so far, but there are significant epidemiologic variations by species such as differences in risk factors and bacterial geographic distribution. ${ }^{1-4}$

The incidence of NTM infections is growing worldwide including in the Middle East countries. ${ }^{5}$ Determining the epidemiology of NTM infection has been more challenging than for TB. Reporting NTM infection to public health authorities is
Correspondence: Abolfazl Fateh Department of Mycobacteriology and Pulmonary Research, Pasteur Institute of Iran, Tehran I 3 1694355 I, Iran

Tel +982164112282

Fax +98216411 2213

Email afateh2@gmail.com 
not compulsory in most parts of the world in comparison with TB reports. Therefore, there is no accurate epidemiological information and monitoring, ${ }^{6-8}$ thus reducing our knowledge and understanding of the effect of NTM infections on community health. ${ }^{5}$ This eventually culminates in mycobacterial infections and their spread across healthcare units. ${ }^{9}$ Understanding the trends and determining the real prevalence of NTM infection is an important priority for optimizing infection control programs and resources. ${ }^{10}$ Several studies have indicated isolation of NTM infection from both TB-suspected patients and the general public in some parts of the country. ${ }^{7,11}$ However, the prevalence of NTM infection has still remained largely unknown. Therefore, the aim of the current study was to evaluate the prevalence of NTM infection among respiratory samples and to determine their clinical importance.

\section{Materials and methods}

\section{Sample collection and preparation}

This was a cross-sectional study conducted from December 2015 to December 2017 on a total of 7,825 TB-suspected respiratory clinical samples referring to Pasteur Institute of Iran. NTM isolates were identified using the guidelines by the American Thoracic Society and Infectious Diseases Society of America (ATS/IDSA). ${ }^{12}$ We included patients with a minimum of two positive cultures from sputum samples and/or at least one positive culture from bronchoalveolar lavage (BAL), samples were considered as clinically relevant to define NTM pulmonary disease (Figure 1). The exclusion criteria were non-respiratory samples such as skin, urine, pus, joints, lymph node and soft tissues and Mycobacterium tuberculosis complex (MTC) positive samples.

This study was in accordance with the Declaration of Helsinki (1975) and local regulations. It was also approved by institutional review boards at Pasteur Institute of Iran (IR.PII.REC.1394.54). Written informed consents were received from all individual. All samples were decontaminated using $\mathrm{N}$-acetyl-L-cysteine-sodium hydroxide method and were cultured on three slopes of Löwenstein-Jensen (LJ) medium. ${ }^{13}$

\section{Identification of NTM strains using phenotypic and genotypic tests}

The phenotypic tests for isolation of NTM strains included macroscopic and microscopic morphological characteristics, growth rate on $\mathrm{LJ}$ medium, growth at temperature of $25^{\circ} \mathrm{C}$, $32^{\circ} \mathrm{C}, 37^{\circ} \mathrm{C}$, and $42^{\circ} \mathrm{C}$, Tween- 80 hydrolysis, arylsulfatase, urease production, tellurite reduction, nitrate reduction, semiquantitative catalase production, and salt tolerance, according to the Centers for Disease Control (CDC) procedures. ${ }^{13}$

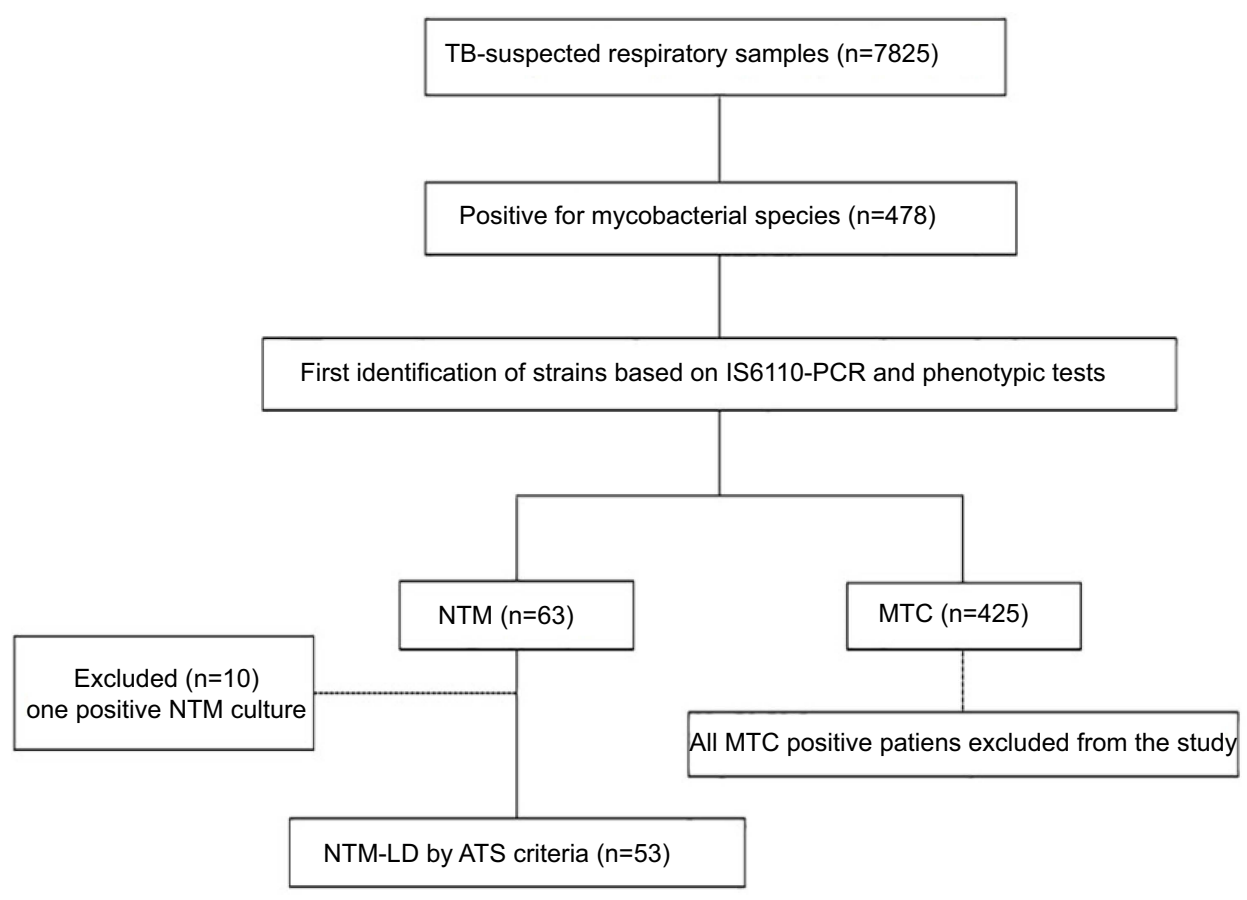

Figure I Population study flowchart.

Abbreviations: TB, tuberculosis; MTC, Mycobacterium tuberculosis complex; NTM-LD, nontuberculous mycobacteria lung disease; ATS, American Thoracic Society. 
Bacterial DNA was isolated using Proba-NK DNA extraction kit (DNA-Technology Company, Moscow, Russia), according to the manufacturer's instructions. The insertion sequence 6110 (IS6110)-PCR (123 bp) method was applied for differentiation of MTC and NTM species in positive cultures. ${ }^{14}$

All NTM strains were primarily detected by using 441 bp fragment of heat shock protein 65 (hsp65), followed by restriction fragment length polymorphism (RFLP) via BstEII and HaeIII restriction enzymes. ${ }^{15}$ Also, NTM isolates detected only up to Mycobacterial species level were investigated for sequencing two highly conserved genes, $16 S$ rRNA (nearly 1,500 bp) and rpoB (750 bp) genes, ${ }^{16,17}$ using an ABI Automated Sequencer (Applied Biosystems, Foster City, CA, USA).

\section{Drug susceptibility testing (DST)}

The minimum inhibitory concentrations (MICs) of each drug were performed by the broth microdilution method and interpreted in accordance with the guidelines from the Clinical and Laboratory Standards Institute (CLSI). ${ }^{18,19}$ The drugs tested included isoniazid, rifampicin, ethambutol, streptomycin, ethionamide, amikacin, ofloxacin, ciprofloxacin, and capreomycin. Serial double dilutions of drugs were prepared ranging from 0.06 to $512 \mathrm{mg} / \mathrm{L}$, added to cationadjusted Mueller-Hinton broth with the addition of 5\% oleic acid-albumin-dextrose-catalase. After inoculating, all cultures were incubated aerobically at $37^{\circ} \mathrm{C}$. Growth was evaluated on days 3 and 7 for rapidly growingmycobacteria (RGM) and weekly up to 4 weeks for slowly growingmycobacteria (SGM). The MIC was defined as the lowest concentration of the antimicrobial agents that inhibited visible growth. Susceptible, moderately susceptible and resistant breakpoints were assigned according to the CLSI guidelines. ${ }^{18}$

\section{Statistical analysis}

All clinical data and demographic characteristics were evaluated by using SPSS version 24.0 (2016; IBM Corp., Armonk, NY, USA) software package. Two-tailed $P$-value $<0.05$ was considered statistically significant. Data normality of continuous variables was initially verified using the Shapiro-Wilk test. Fisher's exact test $/ \chi^{2}$ and MannWhitney $U$-test were used to determine significant association between qualitative and continuous variables, respectively. Continuously distributed variables were described by reporting their mean.

\section{Results}

\section{Patient's characteristics}

A total of 7,825 TB-suspected respiratory samples were included in this study. According to the culture method, $478(6.1 \%)$ samples yielded acid-fast bacilli on culture. Employing phenotypic and molecular methods, 425 (88.9\%) and 53 (11.1\%) subjects were MTC and NTM strains, respectively.

The baseline demographic characteristics of the NTM patients are summarized in Table 1. Briefly, all samples were from pulmonary site including 45 (84.9\%) sputum and eight (15.1\%) BAL. Cough, sputum, fever, weight loss, and night perspiration were the most frequent symptoms. The NTM patient's average age was $43.4 \pm 15.7$ years, and $28(52.8 \%)$ and $25(47.2 \%)$ patients were male and female, respectively. In addition, 8 (15.1\%), 6 (11.3\%), and 5 (9.4\%) cases had HIV, cystic fibrosis (CF) and, diabetes mellitus, respectively.

\section{Phenotypic tests for detection of NTM isolates}

Out of 53 isolates, 33 (62.3\%) and 20 (37.7\%) NTM isolates were RGM and SGM, respectively. According to the phenotypic tests, M. fortuitum (29 isolates) and M. simiae (9 isolates) were the most frequent strains, followed by M. kansasii (4 isolates) and M. gordonae (2 isolates). Among 53 isolates, only 45 (84.9\%) strains were identified by phenotypic tests, while the rest of isolates were unidentifiable.

\section{Molecular tests for detection of NTM isolates}

According to molecular tests ( $h s p 65$-RFLP, rpoB, and $16 S$ $r R N A$ ), NTM isolates included $M$. fortuitum (31 isolates), $M$. simiae (12 isolates), M. kansasii (6 isolates), M. gordonae ( 2 isolates), and M. conceptionense (2 isolates) (Table 1).

\section{Clinical importance of NTM isolates}

The patients' characteristics according to NTM isolates are reported in Table 2. Briefly, there were no significant associations between NTM isolates and the mean age, history of smoking, presence of underlying disease, sample location, and smear microscopy results. However, there was a relationship between NTM isolates with gender, symptoms and radiographic findings. In the $M$. fortuitum positive group, AFB smears were positive for $25.8 \%$ of cases. Also, bronchiectasis $(54.8 \%)$ was the most frequent chest radiography results. Here, 6 (19.4\%), 4 (12.9\%), and 2 (6.5\%) 
Table I Clinical characteristic of patients infected with NTM isolates

\begin{tabular}{|c|c|}
\hline Factors & NTM positive patients $(n=53)$ \\
\hline Mean age \pm SD & $52.9 \pm 13.8$ \\
\hline \multicolumn{2}{|l|}{ Gender } \\
\hline Male & $28(52.8 \%)$ \\
\hline Female & 25 (47.2\%) \\
\hline \multicolumn{2}{|l|}{ History of smoking } \\
\hline Smokers & $16(30.2 \%)$ \\
\hline Non-smokers & 37 (69.8\%) \\
\hline \multicolumn{2}{|l|}{ Symptoms } \\
\hline Cough & 49 (92.5\%) \\
\hline Sputum & 45 (84.9\%) \\
\hline Fever & 45 (84.9\%) \\
\hline Weight loss & $35(66.1 \%)$ \\
\hline Night perspiration & $32(60.4 \%)$ \\
\hline Gastroesophageal & $26(49.1 \%)$ \\
\hline Dyspnea & 18 (33.9\%) \\
\hline Hemoptysis & $12(22.6 \%)$ \\
\hline \multicolumn{2}{|l|}{ Underlying disease } \\
\hline HIV & $8(15.1 \%)$ \\
\hline Cystic fibrosis & $6(11.3 \%)$ \\
\hline Diabetes mellitus & $5(9.4 \%)$ \\
\hline \multicolumn{2}{|l|}{ NTM sample location } \\
\hline Sputum & 45 (84.9\%) \\
\hline Bronchoalveolar lavage & $8(15.1 \%)$ \\
\hline \multicolumn{2}{|c|}{ AFB smear microscopy } \\
\hline Positive & $12(22.6 \%)$ \\
\hline Negative & $4 \mathrm{l}(77.4 \%)$ \\
\hline \multicolumn{2}{|l|}{ Radiographic findings } \\
\hline Bronchiectasis & $26(49.1 \%)$ \\
\hline Infiltrate & $19(35.8 \%)$ \\
\hline Cavitary & $10(18.9 \%)$ \\
\hline Consolidation & $9(17.1 \%)$ \\
\hline \multicolumn{2}{|l|}{ NTM isolates } \\
\hline M. fortuitum & 31 (58.5\%) \\
\hline M. simiae & $12(22.6 \%)$ \\
\hline M. kansasii & 6 (II.3\%) \\
\hline M. gordonae & $2(3.8 \%)$ \\
\hline M. conceptionense & $2(3.8 \%)$ \\
\hline
\end{tabular}

Abbreviations: NTM, nontuberculous mycobacteria; AFB, acid-fast bacilli.

patients had HIV, CF, and diabetes mellitus, respectively, with $38.7 \%$ of patients being smoker. All infected patients had gastroesophageal diseases such as chronic vomiting and achalasia. The major symptoms observed in these patients included cough, sputum, fever, and weight loss (Figure 2). Most of the M. fortuitum strains were resistant to isoniazid, rifampicin, ethambutol, and streptomycin, and susceptible to amikacin (Table S1).

In the M. simiae group, $2(16.7 \%), 1(8.3 \%)$, and 1 (8.3\%) patients had diabetes mellitus, HIV, and CF, respectively. Infiltrate with high frequency was seen in this group. Patients infected with M. simiae had an older mean age and most of them were female (83.3\%). The majority of $M$. simiae strains were resistible to isoniazid, rifampicin, ethambutol, and streptomycin, while susceptible to amikacin, ofloxacin, and ciprofloxacin (Table S2).

In the M. kansasii group, HIV infectivity was shown in one $(1.9 \%)$ patients, and one $(1.9 \%)$ of the patients had diabetes mellitus. All patients had cavitary lesion in their chest radiography results. Hemoptysis was more common in this group than in other patients (Figure 2). Most of the $M$. kansasii strains were susceptible to isoniazid, rifampicin, ethambutol, and streptomycin, while resistant to amikacin, ofloxacin, and ciprofloxacin (Table S2). Infections caused by M. kansasii, M. fortuitum, and M. conceptionense were more frequent in male than in female patients.

\section{Discussion}

NTM pulmonary infections have increasingly been reported in both immunocompetent and immunocompromised individuals worldwide. ${ }^{20-22}$ In several studies, $M$. avium-intracellulare (MAC) infection has been the most common NTM isolates, followed by M. abscessus/chelonae, $M$. fortuitum, and M kansasii. ${ }^{22,23}$ The results of our study indicated that $M$. fortuitum was the most common NTM isolation species, followed by $M$. simiae and $M$. kansasii in Iranian patients.

In Iran, M. fortuitum is the most frequent RGM isolated from both environmental and clinical samples. ${ }^{5}$ Predisposing factors including chronic reflux disease, HIV, malignancy, achalasia, $\mathrm{CF}$, and bronchiectasis in patients infected with $M$. fortuitum usually cause pulmonary disease. ${ }^{24,25}$ The infectious pulmonary disease pattern includes pneumonia, lung abscesses, solitary pulmonary nodules, and pleural effusions. ${ }^{24}$ In line with other studies, most patients had underlying diseases such as HIV, CF, and diabetes mellitus. Also, bronchiectasis was the most common radiographic findings in these patients. M. fortuitum pulmonary infection seems to usually occur in structural lung disease. ${ }^{24}$

According to ATS/IDSA guideline, M. fortuitum was occurred in $15 \%$ of patients with pulmonary disease owing 


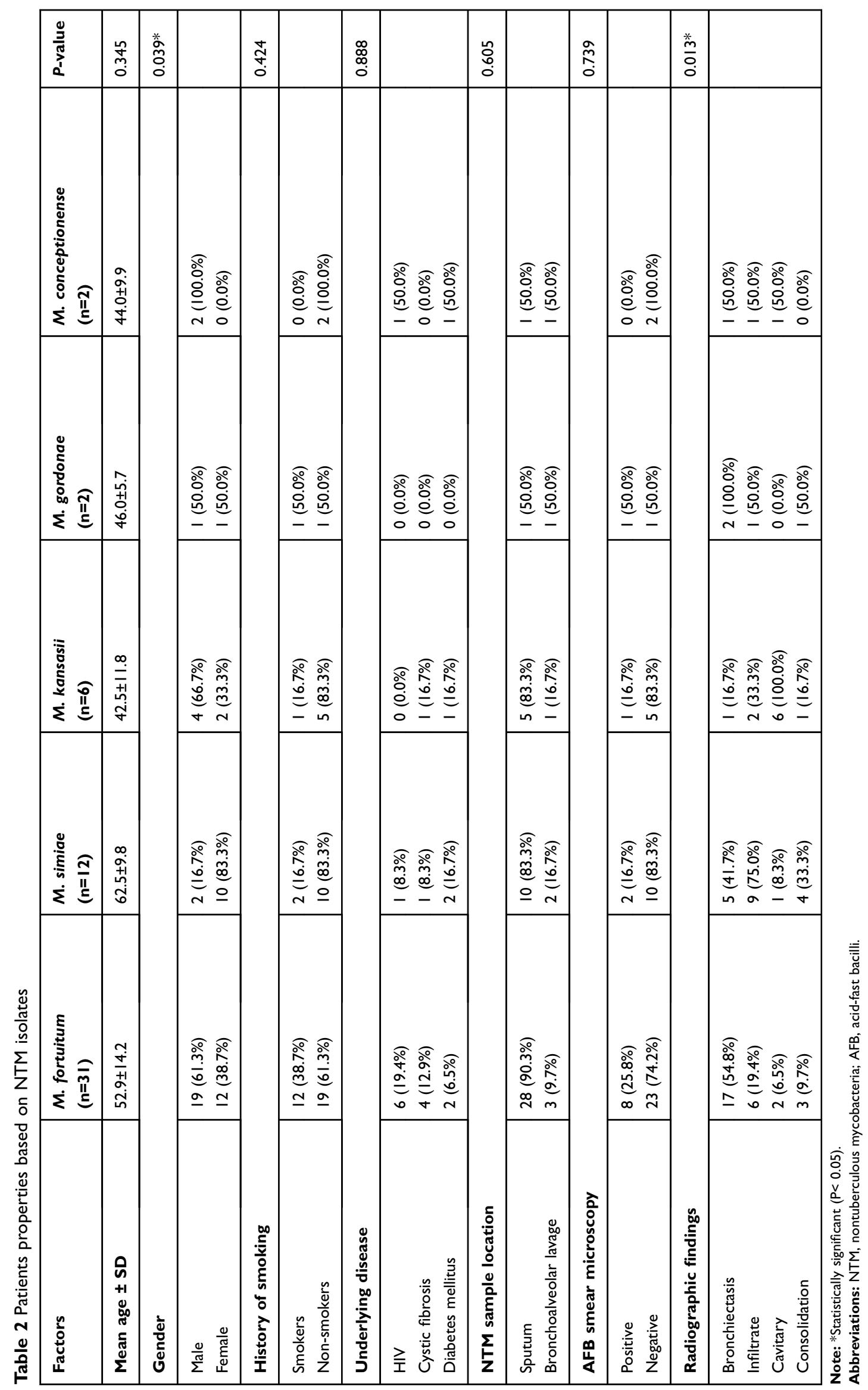




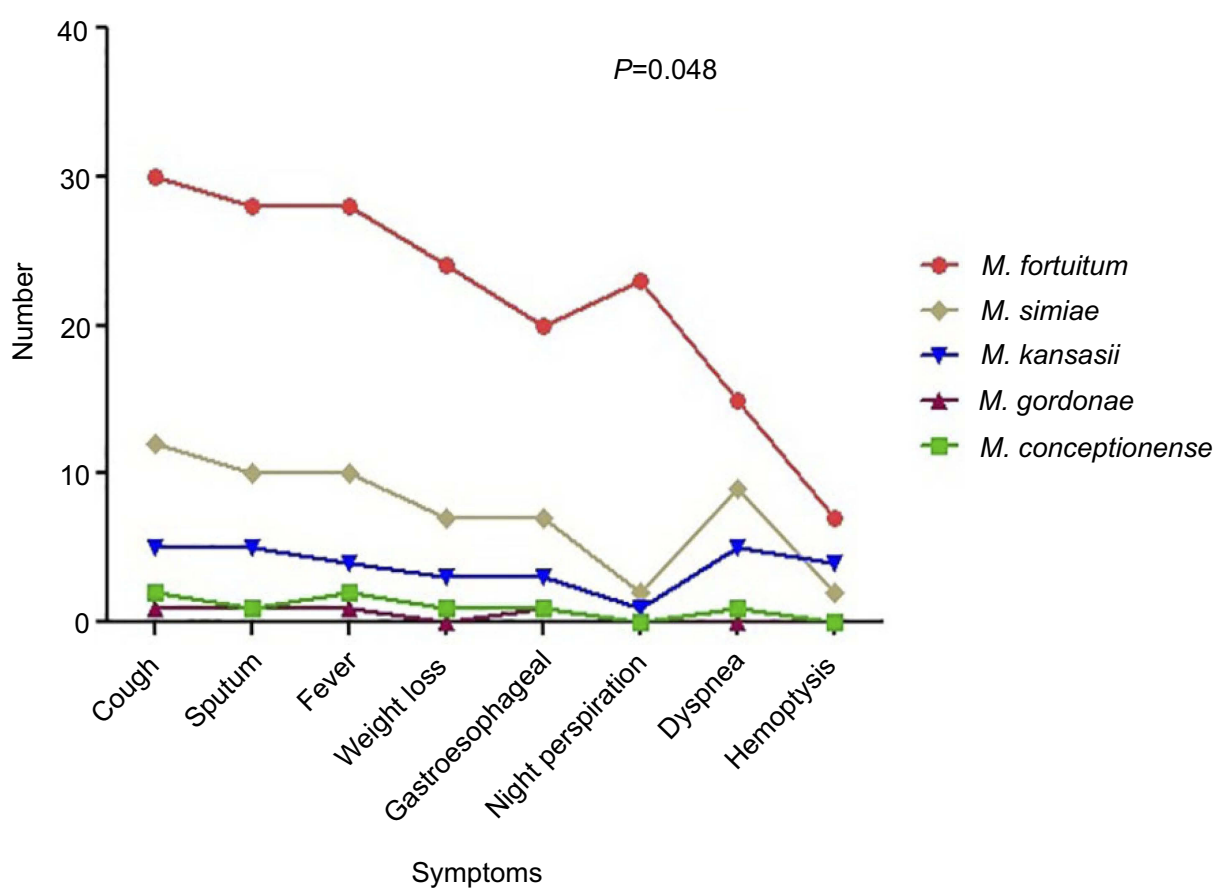

Figure 2 Symptoms of patients infected with nontuberculous mycobacteria (NTM) isolates.

to RGM usually observed as a pathogen in patients with gastroesophageal disease such as chronic vomiting, achalasia, and exogenous lipoid pneumonia. ${ }^{12}$ Studying patients with NTM pulmonary disease as well as esophageal disorders, Hadjiliadis et al revealed that most of them had achalasia and lung infection by $M$. fortuitum. ${ }^{26}$ In the current study, most of $M$. fortuitum infected patients complained about gastroesophageal disease and achalasia, suggests them being an important contributor to $M$. fortuitum pulmonary disease. ${ }^{25}$

All $M$. fortuitum positive patients had two or more positive culture results. Several studies observing $M$. fortuitum isolation in two of the three respiratory sample cultures proposed that although $M$. fortuitum lung disease is rare, this could potentially be the cause of disease. ${ }^{12}$ However, the results of our study have suggested a high pathogenicity of $M$. fortuitum in respiratory samples.

DST results against $M$. fortuitum isolates indicated that this isolate was susceptible to amikacin and intermediate to ofloxacin, ciprofloxacin, and capreomycin in our study. The $M$. fortuitum group is generally more susceptible to drugs than other RGM species. They are usually susceptible or intermediate to doxycycline, fluoroquinolones, sulfonamides, and macrolides. ${ }^{27}$ However, Park et al suggested that long-term treatment with antibiotics may not be necessary for most $M$. fortuitum positive patients from respiratory samples. For this group of patients, infection with $M$. fortuitum can be seen as colonization or a transient infection, and thus less invasive treatment strategies may be indicated. ${ }^{24}$

The second frequent NTM in our study was $M$. simiae. The presence of $M$. simiae in respiratory samples could suggest a real infection or colonization. ${ }^{11}$ The current study indicated that most patients infected with $M$. simiae were female and older than those with other pulmonary NTM isolates. Several studies have revealed that men outnumber women in pulmonary disease caused by all NTM species, except for M. abscessus, M. chelonae, and M. simiae, which is in line with our results. ${ }^{28,29}$

The $M$. simiae positive cases in our study had underlying diseases such as HIV, CF and diabetes mellitus. According to early surveillance reports, $21 \%$ of $M$. simiae patient isolates had underlying disease, although other reports have shown a far lower incidence of clinical disease. $^{12}$ Nevertheless, earlier reports revealed that NTM disease mainly occurs in patients with diabetes, $\mathrm{CF}$, silicosis, and pneumoconiosis. ${ }^{30}$ Although the HIV infection is very important for increased NTM infection, Maoz et al reported that $M$. simiae positive patients were HIV negative. These findings may demonstrate that a clinical suspicion for $M$. simiae infection should also be considered more frequently in non-HIV subjects. ${ }^{28}$ 
M. simiae may have similar clinical and radiologic manifestations with $M$. tuberculosis. ${ }^{11}$ In the current study, M. simiae was isolated from patients previously diagnosed as multidrug-resistant $\mathrm{TB}$, who had received anti-TB drugs. As a result, for every $M$. simiae isolate, DST should be performed. ${ }^{31}$ In our isolated strain, due to financial reasons and lack of access to all drugs, DST was not conducted with all drugs. However, M. simiae isolates from the present study were mainly susceptible to amikacin, ofloxacin, and ciprofloxacin, unlike the findings by Van Ingen et al, which indicated susceptibility rates of 14 $40 \%$ for amikacin and $33-62 \%$ for ciprofloxacin. ${ }^{31}$ These results confirm the variable susceptibly profile of $M$. simiae in various geographic regions, emphasizing the need to do the susceptibility testing before starting treatment. However, there is little information linking in vitro susceptibility to treatment responses. ${ }^{30}$

M. kansasii was the third most frequent pulmonary NTM in the current study. It has clinical and antigenic properties similar to $M$. tuberculosis whose high prevalence has been shown in polluted cities. ${ }^{5,32}$

In this study, radiographic findings for M. kansasii pulmonary disease were diverse. Cavitary lesion was the most frequent radiographic features. Several studies have demonstrated cavitation in $75-96 \%$ of patients with $M$. kansasii pulmonary disease. ${ }^{33,34}$ The radiographic characteristics of $M$. kansasii pulmonary disease are commonly known as being indiscernible from those of M. tuberculosis.

Appropriate treatment regimen for M. kansasii pulmonary disease is a three-drug combination including isoniazid, rifampin, and ethambutol for at least 12 months after negative sputum conversion. In our study, DST for M. kansasii pulmonary disease, in accordance with other studies, had the same results with the treatment regimen. All M. kansasii isolate were resistant to isoniazid, rifampin, and the highest resistance to ciprofloxacin. ${ }^{35}$ In line with our study, Shitrit et al showed that M. kansasii isolates had highest sensitivity to rifampin, ethambutol, and the highest resistance to ciprofloxacin. ${ }^{19}$

Hemoptysis occurred in 4 (66.7\%) of the M. kansasii cases in our group patients, which was higher than the rates of other studies. ${ }^{12,33}$ Hemoptysis in lung infections can be related to the incidence of endobronchial disease and lead to disintegration of bronchial vessels by cavitation. Although there is no practical information on the relative incidence of endobronchial disease, the incidence of cavitation in the M. kansasii pulmonary infection is reported to be $57 \%$, which is slightly close to our study. Note that cavitations were far less common in patients with other NTM infections. ${ }^{34-36}$

We found higher patients with hemoptysis, dyspnea, and cough and fewer patients with fever and night perspiration. This result was inconsistent with the report of Shitrit et $\mathrm{al}^{19}$. These discrepancies may have been owing to the short interval from symptom onset to diagnosis. ${ }^{19}$

In the current study, M. conceptionense was rarely identified in respiratory samples. It was reported that this isolate caused skin and subcutaneous fat infections under surgery in immunocompromised patients. ${ }^{37}$ Furthermore, few studies confirmed that this isolate may be a pulmonary pathogen. ${ }^{38,39}$ In Iran, only one study had revealed $M$. conceptionense in two patients with pulmonary disease. ${ }^{40}$

For the second time in Iran, we identified M. conceptionense in 37-year-old male with HIV infection and 51year-old male with diabetes mellitus. Interestingly, both patients had underlying disease and DST showed susceptibility to ofloxacin, ciprofloxacin, and amikacin as well as intermediate to isoniazid, rifampicin, and ethionamide. These findings were in agreement with the Kim et al's study $^{39}$ suggesting that $M$. conceptionense can cause pulmonary disease, though it is a rare NTM in Iran.

The main limitations of our study were the lack of access to follow-up information. Also, the radiological evaluation focused mainly on chest radiography, not on CT scan.

\section{Conclusion}

We detected five NTM species in respiratory samples. $M$. fortuitum was the most frequent NTM species followed by $M$. simiae and $M$. kansasii with different radiographic findings, clinical symptoms, and drug resistance. M. conceptionense as a rare NTM isolate was reported for the second time in Iran. However, future studies are required to assess the epidemiology of respiratory NTM in Iran to increase the knowledge of Iranian physicians for the diagnosis and treatment of NTM isolates.

\section{Acknowledgments}

The authors would like to thank all the personnel from the Department of Mycobacteriology and Pulmonary Research, Pasteur Institute of Iran.

\section{Disclosure}

The authors report no conflicts of interest in this work. 


\section{References}

1. Adjemian J, Frankland TB, Daida YG, et al. Epidemiology of nontuberculous mycobacterial lung disease and tuberculosis, Hawaii, USA. Emerg Infect Dis. 2017;23(3):439-447. doi:10.3201/eid2303.161827

2. Shah NM, Davidson JA, Anderson LF, et al. Pulmonary Mycobacterium avium-intracellulare is the main driver of the rise in non-tuberculous mycobacteria incidence in England, Wales and Northern Ireland, 2007-2012. BMC Infect Dis. 2016;16(1):195. doi:10.1186/s12879-016-1521-3

3. Adjemian J, Olivier KN, Seitz AE, Holland SM, Prevots DR. Prevalence of nontuberculous mycobacterial lung disease in US Medicare beneficiaries. Am J Respir Crit Care Med. 2012;185 (8):881-886. doi:10.1164/rccm.201111-2016OC

4. Yu X, Liu P, Liu G, et al. The prevalence of non-tuberculous mycobacterial infections in mainland China: systematic review and meta-analysis. $J$ Infect. 2016;73(6):558-567. doi:10.1016/j.jinf.2016.08.020

5. Velayati AA, Rahideh S, Nezhad ZD, Farnia P, Mirsaeidi M. Nontuberculous mycobacteria in Middle East: current situation and future challenges. Int J Mycobacteriol. 2015;4(1):7-17. doi:10.1016/ j.ijmyco.2014.12.005

6. Kendall BA, Winthrop KL. Update on the epidemiology of pulmonary nontuberculous mycobacterial infections. Semin Respir Crit Care Med. 2013;34(1):87-94. doi:10.1055/s-0033-1333567

7. Velayati AA, Farnia P, Mozafari M, et al. Molecular epidemiology of nontuberculous mycobacteria isolates from clinical and environmental sources of a metropolitan city. PLoS One. 2014;9(12):e114428. doi:10.1371/journal.pone.0114428

8. Yoon HJ, Choi HY, Ki M. Nontuberculosis mycobacterial infections at a specialized tuberculosis treatment centre in the Republic of Korea. BMC Infect Dis. 2017;17(1):432. doi:10.1186/s12879-0172532-4

9. Sousa S, Bandeira M, Carvalho PA, Duarte A, Jordao L. Nontuberculous mycobacteria pathogenesis and biofilm assembly. Int J Mycobacteriol. 2015;4(1):36-43. doi:10.1016/j.jijmyco.2014.11.065

10. Gopinath K, Singh S. Non-tuberculous mycobacteria in TB-endemic countries: are we neglecting the danger? PLoS Negl Trop Dis. 2010;4 (4):e615. doi:10.1371/journal.pntd.0000615

11. Nasiri MJ, Dabiri H, Fooladi AAI, Amini S, Hamzehloo G, Feizabadi MM. High rates of nontuberculous mycobacteria isolation from patients with presumptive tuberculosis in Iran. New Microbes New Infect. 2018;21:12-17. doi:10.1016/j.nmni.2017.08.008

12. Griffith DE, Aksamit T, Brown-Elliott BA, et al. An official ATS/ IDSA statement: diagnosis, treatment, and prevention of nontuberculous mycobacterial diseases. Am J Respir Crit Care Med. 2007;175 (4):367-416. doi:10.1164/rccm.200604-571ST

13. Kent PT, Kubica GP. Public Health Mycobacteriology: A Guide for the Level III Laboratory. U.S. Department of Health and Human Services, Public Health Service, Centers for Disease Control and Prevention, Atlanta, GA; 1985.

14. Eisenach KD, Donald Cave M, Bates JH, Crawford JT. Polymerase chain reaction amplification of a repetitive DNA sequence specific for Mycobacterium tuberculosis. J Infect Dis. 1990;161(5):977-981. doi:10.1093/infdis/161.5.977

15. Telenti A, Marchesi F, Balz M, Bally F, Böttger E, Bodmer T. Rapid identification of Mycobacteria to the species level by polymerase chain reaction and restriction enzyme analysis. J Clin Microbiol. 1993;31(2):175-178.

16. Rogall T, Flohr T, Böttger EC. Differentiation of Mycobacterium species by direct sequencing of amplified DNA. J Gen Microbiol. 1990;136(9):1915-1920. doi:10.1099/00221287-136-9-1915

17. Adékambi T, Colson P, Drancourt M. rpoB-based identification of nonpigmented and late-pigmenting rapidly growing mycobacteria. $J$ Clin Microbiol. 2003;41(12):5699-5708. doi:10.1128/JCM.41.12.56995708.2003
18. CLSI. Susceptibility testing of Mycobacteria, Nocardiae, and other Aerobic Actinomycetes; approved standard-second edition. CLSI document M24-A2. Wayne (PA): Clinical and Labo-ratory Standards Institute; 2011.

19. Shitrit D, Baum GL, Priess R, et al. Pulmonary Mycobacterium kansasii infection in Israel, 1999-2004: clinical features, drug susceptibility, and outcome. Chest. 2006;129(3):771-776. doi:10.1378/ chest.129.3.771

20. Adjemian J, Daniel-Wayman S, Ricotta E, Prevots DR. Epidemiology of nontuberculous mycobacteriosis. Semin Respir Crit Care Med. 2018;39(3):325-335. doi:10.1055/s-0038-1651491

21. Andréjak C, Thomsen VØ, Johansen IS, et al. Nontuberculous pulmonary mycobacteriosis in Denmark: incidence and prognostic factors. Am J Respir Crit Care Med. 2010;181(5):514-521. doi:10.1164/ rccm.200905-0778OC

22. Duan H, Han X, Wang Q, et al. Clinical significance of nontuberculous mycobacteria isolated from respiratory specimens in a Chinese tuberculosis tertiary care center. Sci Rep. 2016;6:36299. doi:10.1038/ srep36299

23. Prevots DR, Marras TK. Epidemiology of human pulmonary infection with nontuberculous mycobacteria: a review. Clin Chest Med. 2015;36(1):13-34. doi:10.1016/j.ccm.2014.10.002

24. Park S, Suh GY, Chung MP, et al. Clinical significance of Mycobacterium fortuitum isolated from respiratory specimens. Respir Med. 2008;102(3):437-442. doi:10.1016/j.rmed.2007.10.005

25. Irandoost M, Zare Ghanbari M, Sakhaee F, et al. High rates of Mycobacterium fortuitum isolation in respiratory samples from Iranian patients with suspected tuberculosis: is it clinically important? J Med Microbiol. 2018;67(9):1243-1248. doi:10.1099/jmm.0.000814

26. Hadjiliadls D, Adlakha A, Prakash UB. Rapidly growing mycobacterial lung infection in association with esophageal disorders. Mayo Clin Proc. 1999;74(1):45-51. doi:10.4065/74.1.45

27. Brown-Elliott BA, Philley JV. Rapidly growing mycobacteria. Microbiol Spectr. 2017;5(1). doi:10.1128/microbiolspec.TNMI7-0027-2016

28. Maoz C, Shitrit D, Samra Z, et al. Pulmonary Mycobacterium simiae infection: comparison with pulmonary tuberculosis. Eur J Clin Microbiol Infect Dis. 2008;27(10):945-950. doi:10.1007/s10096008-0522-6

29. Van Ingen J, Boeree M, Dekhuijzen P, Van Soolingen D. Clinical relevance of Mycobacterium simiae in pulmonary samples. Eur Respir J. 2008;31(1):106-109. doi:10.1183/09031936.00076107

30. Hamieh A, Tayyar R, Tabaja H, et al. Emergence of Mycobacterium simiae: a retrospective study from a tertiary care center in Lebanon. PLoS One. 2018;13(4):e0195390. doi:10.1371/journal.pone.0195390

31. van Ingen J, Totten SE, Heifets LB, Boeree MJ, Daley CL. Drug susceptibility testing and pharmacokinetics question current treatment regimens in Mycobacterium simiae complex disease. Int J Antimicrob Agents. 2012;39(2):173-176. doi:10.1016/j.ijantimicag.2011.09.019

32. Nour-Neamatollahie A, Ebrahimzadeh N, Siadat SD, et al. Distribution of non-tuberculosis mycobacteria strains from suspected tuberculosis patients by heat shock protein 65 PCR-RFLP. Saudi $J$ Biol Sci. 2017;24(6):1380-1386. doi:10.1016/j.sjbs.2016.02.001

33. Matveychuk A, Fuks L, Priess R, Hahim I, Shitrit D. Clinical and radiological features of Mycobacterium kansasii and other NTM infections. Respir Med. 2012;106(10):1472-1477. doi:10.1016/j.rmed.2012.06.023

34. Shitrit D, Peled N, Bishara J, et al. Clinical and radiological features of Mycobacterium kansasii infection and Mycobacterium simiae infection. Respir Med. 2008;102(11):1598-1603. doi:10.1016/j. rmed.2008.05.004

35. Davari M, Irandoost M, Sakhaee F, et al. Genetic diversity and prevalence of nontuberculous mycobacteria isolated from clinical samples in Tehran, Iran. Microb Drug Resist. 2019;25(2):264-270. doi: $10.1089 / \mathrm{mdr} .2018 .0150$

36. Park HK, Koh W-J, Shim TS, Kwon OJ. Clinical characteristics and treatment outcomes of Mycobacterium kansasii lung disease in Korea. Yonsei Med J. 2010;51(4):552-556. doi:10.3349/ymj.2010.51.4.552 
37. Lee KH, Heo ST, Choi SW, Park DH, Kim YR, Yoo SJ. Three cases of postoperative septic arthritis caused by Mycobacterium conceptionense in shoulder joints of immunocompetent patients. J Clin Microbiol. 2014;1013-1015. doi:10.1128/JCM.02652-13

38. Kim J, Seong MW, Kim EC, Han SK, Yim JJ. Frequency and clinical implications of the isolation of rare nontuberculous mycobacteria. BMC Infect Dis. 2015;15(1):9. doi:10.1186/s12879-014$0741-7$
39. Kim SY, Kim MS, Chang HE, et al. Pulmonary infection caused by Mycobacterium conceptionense. Emerg Infect Dis. 2012;18(1):174176. doi:10.3201/eid1801.110251

40. Shojaei H, Hashemi A, Heidarieh P, Ataei B, Naser AD. Pulmonary and extrapulmonary infection caused by Mycobacterium conceptionense: the first report from Iran. JRSM Short Rep. 2011;2(4):31. doi:10.1258/shorts.2010.010103 


\section{Supplementary materials}

Table SI Antimycobacterial susceptibility testing results for clinical isolates of RGM

\begin{tabular}{|c|c|c|c|c|c|c|}
\hline \multirow[t]{2}{*}{ Bacteria (no.) and antimicrobial agent } & \multirow[b]{2}{*}{ Range } & \multicolumn{2}{|c|}{ MIC $(\mu \mathrm{g} / \mathrm{mL})$} & \multicolumn{3}{|c|}{ No. (\%) of isolates } \\
\hline & & $50 \%$ & $90 \%$ & Susceptible & Intermediate & Resistant \\
\hline \multicolumn{7}{|l|}{ M. fortuitum (3I) } \\
\hline Isoniazid & $2->256$ & $>256$ & $>256$ & $0(0.0 \%)$ & $0(0.0 \%)$ & $31(100.0 \%)$ \\
\hline Rifampicin & $\mathrm{I}->256$ & 128 & $>256$ & $0(0.0 \%)$ & $0(0.0 \%)$ & $31(100.0 \%)$ \\
\hline Ethambutol & $32->256$ & 256 & $>256$ & $0(0.0 \%)$ & $0(0.0 \%)$ & $31(100.0 \%)$ \\
\hline Streptomycin & $8->256$ & 32 & 128 & $0(0.0 \%)$ & $0(0.0 \%)$ & $31(100.0 \%)$ \\
\hline Amikacin & $0.06-16$ & 1 & 8 & $28(90.3 \%)$ & $2(6.5 \%)$ & I (3.2\%) \\
\hline Ofloxacin & $0.06-32$ & 2 & 8 & $15(48.4 \%)$ & 4 (I2.9\%) & $12(38.7 \%)$ \\
\hline Ciprofloxacin & $0.06-8$ & 0.25 & 2 & $15(48.4 \%)$ & $4(12.9 \%)$ & $12(38.7 \%)$ \\
\hline Capreomycin & $0.03-2$ & 0.5 & 2 & $14(45.2 \%)$ & $7(22.6 \%)$ & $10(32.2 \%)$ \\
\hline \multicolumn{7}{|l|}{ M. conceptionense (2) } \\
\hline Isoniazid & $16->256$ & $>256$ & $>256$ & $0(0.0 \%)$ & I (50.0\%) & I (50.0\%) \\
\hline Rifampicin & $2-8$ & 1 & 8 & $0(0.0 \%)$ & I (50.0\%) & I (50.0\%) \\
\hline Ethambutol & $128->256$ & $>256$ & $>256$ & $0(0.0 \%)$ & $0(0.0 \%)$ & $2(100.0 \%)$ \\
\hline Streptomycin & $4-64$ & 32 & 64 & $0(0.0 \%)$ & $0(0.0 \%)$ & $2(100.0 \%)$ \\
\hline Amikacin & $0.25->32$ & 16 & 64 & $2(100.0 \%)$ & $0(0.0 \%)$ & $0(0.0 \%)$ \\
\hline Ofloxacin & $0.03-8$ & 0.5 & 8 & $2(100.0 \%)$ & $0(0.0 \%)$ & $0(0.0 \%)$ \\
\hline Ciprofloxacin & $0.13-8$ & 2 & 4 & $2(100.0 \%)$ & $0(0.0 \%)$ & $0(0.0 \%)$ \\
\hline Capreomycin & $0.03-32$ & 2 & 16 & I (50.0\%) & I (50.0\%) & $0(0.0 \%)$ \\
\hline
\end{tabular}

Abbreviations: MIC, minimum inhibitory concentrations; RGM, rapid-growing mycobacteria. 
Table S2 Antimycobacterial susceptibility testing results for clinical isolates of SGM

\begin{tabular}{|c|c|c|c|c|c|c|}
\hline \multirow[t]{2}{*}{ Bacteria (no.) and antimicrobial agent } & \multirow[b]{2}{*}{ Range } & \multicolumn{2}{|c|}{ MIC $(\mu \mathrm{g} / \mathrm{mL})$} & \multicolumn{3}{|c|}{ No. (\%) of isolates } \\
\hline & & $50 \%$ & $90 \%$ & Susceptible & Intermediate & Resistant \\
\hline \multicolumn{7}{|l|}{ M. simiae (12) } \\
\hline Isoniazid & $16-128$ & 32 & 64 & $0(0.0 \%)$ & $0(0.0 \%)$ & $12(100.0 \%)$ \\
\hline Rifampicin & $0.5-128$ & 2 & 8 & I (8.3\%) & $0(0.0 \%)$ & II(9I.7\%) \\
\hline Ethambutol & $4-64$ & 16 & 64 & $3(25.0 \%)$ & $0(0.0 \%)$ & $9(75.0 \%)$ \\
\hline Streptomycin & $2-64$ & 16 & 32 & $2(16.7 \%)$ & $0(0.0 \%)$ & $10(83.3 \%)$ \\
\hline Amikacin & $0.06-64$ & 2 & 16 & $10(83.3 \%)$ & $0(0.0 \%)$ & $2(16.7 \%)$ \\
\hline Ofloxacin & $2-64$ & 8 & 32 & I (I6.7\%) & $0(0.0 \%)$ & $10(83.3 \%)$ \\
\hline Ciprofloxacin & $0.25-64$ & 8 & 32 & $3(25.0 \%)$ & $0(0.0 \%)$ & 9 (75.0\%) \\
\hline \multicolumn{7}{|l|}{ M. kansasii (6) } \\
\hline Isoniazid & $0.25-16$ & 2 & 4 & $6(100.0 \%)$ & $0(0.0 \%)$ & $0(0.0 \%)$ \\
\hline Rifampicin & $0.125-16$ & 2 & 8 & $6(100.0 \%)$ & $0(0.0 \%)$ & $0(0.0 \%)$ \\
\hline Ethambutol & $0.25-64$ & 2 & 4 & 5 (83.3\%) & $0(0.0 \%)$ & I (16.7\%) \\
\hline Streptomycin & $0.5-32$ & 8 & 32 & $4(66.7 \%)$ & $0(0.0 \%)$ & $2(33.3 \%)$ \\
\hline Amikacin & $0.125-32$ & 2 & 16 & I (I6.7\%) & $0(0.0 \%)$ & $5(83.3 \%)$ \\
\hline Ofloxacin & $0.25-4$ & I & 2 & 2 (33.3\%) & $0(0.0 \%)$ & $4(66.7 \%)$ \\
\hline Ciprofloxacin & $0.25-16$ & 2 & 4 & $2(33.3 \%)$ & $0(0.0 \%)$ & $4(66.7 \%)$ \\
\hline \multicolumn{7}{|l|}{ M. gordonae (2) } \\
\hline Isoniazid & $0.5->256$ & 2 & $>256$ & I (50.0\%) & $0(0.0 \%)$ & I (50.0\%) \\
\hline Rifampicin & $0.125-16$ & 0.25 & 8 & $0(0.0 \%)$ & $0(0.0 \%)$ & $2(100.0 \%)$ \\
\hline Ethambutol & $0.5-64$ & I & 16 & I (50.0\%) & $0(0.0 \%)$ & I (50.0\%) \\
\hline Streptomycin & $0.25-32$ & 1 & 8 & I (50.0\%) & $0(0.0 \%)$ & I (50.0\%) \\
\hline Amikacin & $0.5-128$ & 2 & 32 & $0(0.0 \%)$ & $0(0.0 \%)$ & $2(100.0 \%)$ \\
\hline Ofloxacin & $\mathrm{I}-32$ & 2 & 16 & I (50.0\%) & $0(0.0 \%)$ & I (50.0\%) \\
\hline Ciprofloxacin & $0.125-16$ & 0.5 & 8 & $0(0.0 \%)$ & $0(0.0 \%)$ & $2(100.0 \%)$ \\
\hline
\end{tabular}

Abbreviations: MIC, minimum inhibitory concentrations; SGM, slow-growing mycobacteria.

Infection and Drug Resistance

\section{Publish your work in this journal}

Infection and Drug Resistance is an international, peer-reviewed openaccess journal that focuses on the optimal treatment of infection (bacterial, fungal and viral) and the development and institution of preventive strategies to minimize the development and spread of resistance. The journal is specifically concerned with the epidemiology of antibiotic resistance and the mechanisms of resistance development and diffusion in both hospitals and the community. The manuscript management system is completely online and includes a very quick and fair peerreview system, which is all easy to use. Visit http://www.dovepress.com/ testimonials.php to read real quotes from published authors. 